\title{
A Successful Percutaneous Dilatational Tracheostomy Procedure in Pneumonectomized Patient: A Case Report
}

\author{
Mungan I*, Bektas S, Dicle CB, Kazanci D and Turan S \\ Turkey Advanced Speciality Education and Research Hospital, Department of Intensive Care Unit, Altındag/Ankara, Turkey
}

*Corresponding author: Mungan I, Turkey Advanced Speciality Education and Research Hospital, Department of Intensive Care Unit, Altındag/Ankara, Turkey, Tel: +90-5075081594, E-mail: imungan@gmail.com

Citation: Mungan I, Bektas S, Dicle CB, Kazanci D, Turan S (2018) A successful percutaneous dilatational tracheostomy procedure in pneumonectomized patient: A case report. SAJ Case Rep 5: 302

Article history: Received: 07 May 2018, Accepted: 03 July 2018, Published: 05 July 2018

\begin{abstract}
Tuberculosis (TB) continues to be a substantial cause of morbidity and the current approach to TB treatment is medical chemotherapy. However the most common benign cause of pneumonectomy is still pulmonary tuberculosis and after pneumonectomy mediastinal structures could be displaced resulting airway obstruction. The tracheostomy procedure is recommended for ventilator dependent patients Percutaneous dilatational tracheostomy (PDT) mostly replaced surgical one due to its simplicity and cost effectiveness. In this case report, the authors describe a successful percutaneous dilatational tracheostomy procedure in a pneumonectomized patient.

Case Report: A 60-year-old male who had undergone right pneumonectomy 20 years ago, could not be weaned from mechanical ventilation for a long period and percutaneous dilatational tracheostomy (PDT) procedure was performed successfully. He was weaned from the tracheostomy after 20 days and was discharged after 4 weeks.

Discussion: Surgery is increasingly used in the era of "multi-drug resistant" tuberculosis and pneumonectomy causes displacement of respiratory anatomical structures.The most common cause of the tracheostomy requirement in the ICU is prolonged ventilatory support and in recent years we have seen extensive use of PDT almost supersedes surgical tracheostomy. The choice is often determined by subjective factors, experience, economic issues, and availability. In our case, with the aid of bronchoscopic guidance, PDT was done without any complication but one should not ignore relative contraindications of PDT.
\end{abstract}

Keywords: Percutaneous Dilatational Tracheostomy; Pneumonectomy; Tuberculosis

\section{Introduction}

Tuberculosis (TB) continues to be a substantial cause of morbidity in spite of available medical treatment for more than 65 years [1]. The most common benign cause of pneumonectomy and lung lobectomy is still pulmonary tuberculosis worldwide. After pneumonectomy mediastinal structures could be displaced by sliding towards the gap formed between main bronchus and trachea and this may result in airway obstruction [2].

The tracheostomy procedure is recommended for patients who resumed to be mechanical ventilator dependent for a long time. Mainly two types of tracheostomy are used in the Intensive Care Unit (ICU) practice; Open surgical tracheostomy (OST) and Percutaneous dilatational tracheostomy (PDT) [3]. PDT mostly replaced OST due to its simplicity and cost effectiveness.

OST usage gained popularity in the ICU during the polio epidemic in the 1950's and PDT was the modified version of one stage dilatation technique as described by Griggs et al. in 1990 [3,4]. The technique is to hole the trachea and insert a guide wire into it. This was done blindly until 1990 when Marelli et al. used bronchoscopy as an auxiliary to PDT to increase the safety of the procedure. In this case report, the authors describe a successful percutaneous dilatational tracheostomy procedure in a pneumonectomized patient which is rare in the literature due to its indication [5].

\section{Case report}

A 60-year-old,74-kg,166-cm male who had undergone right pneumonectomy 20 years ago for pulmonary TB was admitted to the coronary intensive care unit for pericardial effusion and pneumonia. He was found ( -) for TB screening tests (the patient's sputum smear results are negative for acid-fast bacilli). The pericardial effusion problem was solved via anterior subxiphoidal 
pericardiostomy procedure and appropriate antibiotic treatment was started empirically after the initial blood and sputum samples for culture were obtained.

He had been supported with non-invasive mechanical ventilation to treat acute respiratory failure that developed 24 hours after admission, but unfortunately the desired appropriate levels of blood gas analysis could not be achieved and he was intubated. After understanding that the patient could not be weaned from mechanical ventilation for a long period and percutaneous dilatational tracheostomy (PDT) procedure was decided to perform. Not only in aspect of experience and affinity but also due to less invasivity and cost effectiveness, the Griggs technique of PDT with bronchoscopic guidance was chosen in our department. The procedure was applied in the ICU under aseptic preventions. The patient was monitored for airway security, ventilation, ECG derives, blood pressure, and $\mathrm{SpO}_{2}$ regularly.

Lidocaine $2 \%$ for local anesthesia and appropriate sedation were used during the procedure. With the aid of the bronchoscope insertion site was visualized and a 14 Gauze cannula was inserted between the second and third tracheal ring at the midline. After entry into the trachea the guide wire, the cannula, the 14-French plastic dilator, metal tracheal dilator forceps and then the tracheostomy tube (TT) was inserted in a stepwise manner. In the whole process bronchoscopic visualization was maintained properly. TTs position was confirmed by bronchoscopy and by chest auscultation. After the procedure antero-posterior chest X-ray confirmed TT place and no complication was detected. Figure 1 and Figure 2 showed AP Chest X-ray before and after the tracheostomy procedure. 2 weeks later, he was off the ventilator and was successfully weaned from the tracheostomy after 20 days and was discharged after 4 weeks.

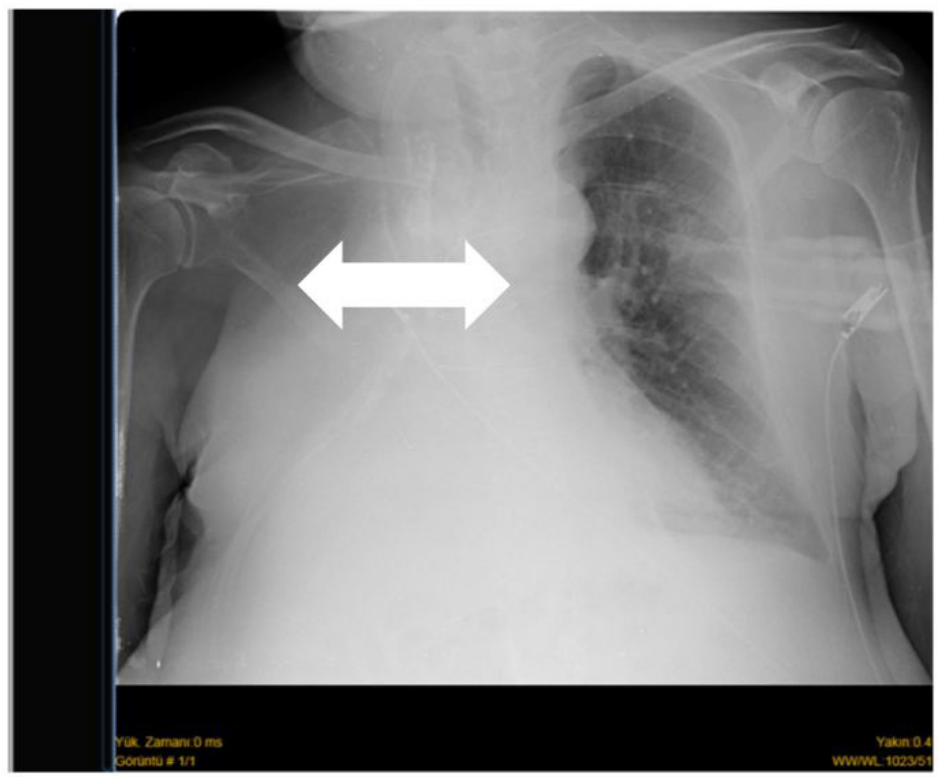

Figure 1: Chest X-ray anterior- posterior view shows previous pneumonectomy with ipsilateral mediastinal shift (indicated by an arrow)

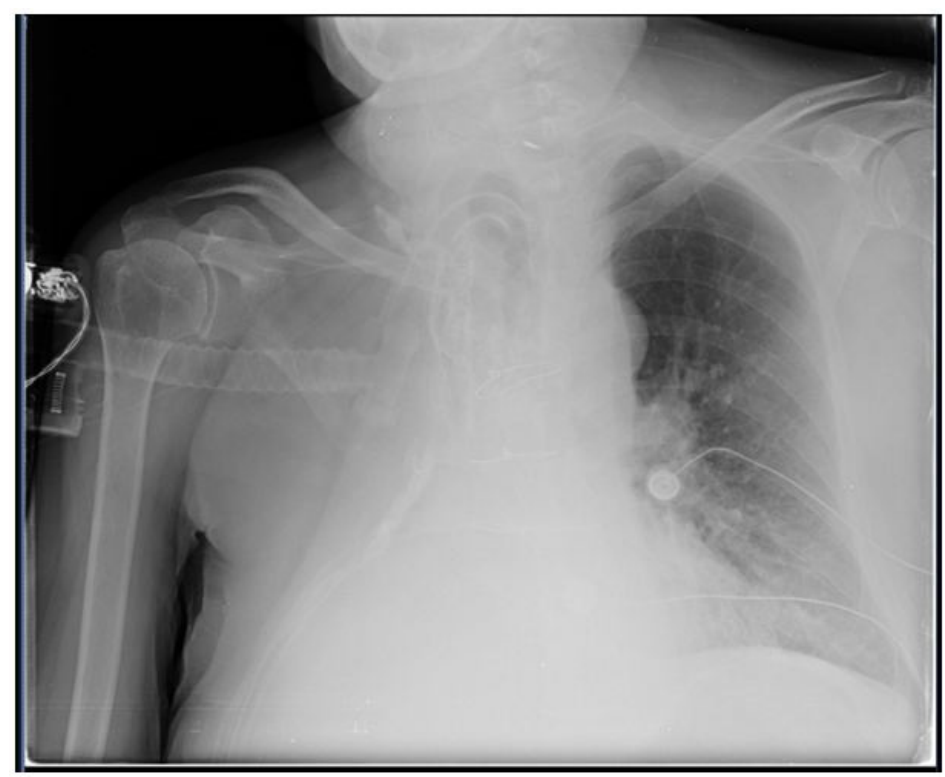

Figure 2: Chest X-ray anterior -posterior view shows successful tracheostomy without any complication 


\section{Discussion}

World Health Organization announced the incidence of tuberculosis in Turkey is 18 per 100,000 population per year in 2016 and the global incidence was estimated as 10.4 million [6]. Surgery is increasingly used in the era of "multi-drug resistant" tuberculosis, to treat complications like massive haemoptysis and bronchiectasis. In some studies it is shown that surgical resection with antibiotherapy accomplished approximately $85 \%$ cure rate [7]. Although the most frequent cause of lung resections is malignant diseases, bronchiectasis, necrotizing pneumonia and TB are benign causes that may require surgical intervention [2]. and it.

The most common cause of the tracheostomy requirement in the ICU is prolonged ventilatory support and approximately one fourth of mechanically ventilated patients undergo tracheostomy [8]. It is claimed that tracheostomy has several advantages over endotracheal intubation like decreasing work of breath, easing bronchial secretion clearance, improving patient's comfort, facilitating wean procedures and minimizes sedation requirement in the ICU [9]. In recent years we have seen extensive use of PDT almost supersedes OST. This is subsidiary to the easy application of PDT at patient's bedside with cost effectiveness and avoidance of redundant, high risky transfers to the operation theater [4,10]. Putensen, et al. compared PDT to OST in a meta analysis and found PDT less inflammatory with high technical difficulties, whereas major guidelines suggest PDT as first choice in non-urgent cases [11]. In some cases like infection at the insertion site, presence of pulsatile vessels at the insertion site, operator inexperience OST choice becomes a must and difficult anatomy (short neck, morbid obesity, limited neck extension, local malignancy and tracheal deviation) is known as a relative contraindication [4].

All methods of percutaneous tracheostomy use a modification of the Seldinger technique, and mostly Ciaglia or Griggs technique is the preferred one as a PDT [12]. The choice is often determined by subjective factors, experience, economic issues, and availability [11]. After lobar resection or pneumonectomy the mediastinal structures are displaced in the form of both slip and rotation and in our case tracheal deviation was clearly detected. This may hesitate anyone to choose PDT rather than open surgical method. However, with the aid of bronchoscopic guidance, PDT was done without any complication in our case.

\section{Conclusion}

In conclusion, even in the case of relative contraindications PDT -like pneumonectomy and difficult anatomy-could be performed successfully with bronchoscopy guidance.

\section{References}

1. Evangelopoulos D, Fonseca JD, Waddell SJ (2015) Understanding anti-tuberculosis drug efficacy: rethinking bacterial populations and how we model them. Int J Infect Dis 32: 76-80.

2. Madansein R, Parida S, Padayatchi N, Singh N, Master I, et al. (2015) Surgical treatment of complications of pulmonary tuberculosis, including drug-resistant tuberculosis. Int J Infect Dis 32: 61-7.

3. Pattnaik SK, Ray B, Sinha S (2014) Griggs percutaneous tracheostomy without bronchoscopic guidance is a safe method: A case series of 300 patients in a tertiary care intensive care unit. Indian J Crit Care Med 18:778-82.

4. Mehta C, Mehta Y (2017) Percutaneous Tracheostomy. Ann Card Anaesth 20: 19-25.

5. Marelli D, Paul A, Manolidis S, Walsh G, Odim JN, et al. (1990) Endoscopic guided percutaneous tracheostomy: Early results of a consecutive trial. J Trauma 30: 433-5.

6. World Health Organization (2018) Estimates of TB and MDR-TB burden are produced by WHO in consultation with countries.

7. Man MA, Nicolau D (2012) Surgical treatment to increase the success rate of multidrug-resistant tuberculosis. Eur J Cardiothorac Surg 42: 9-12.

8. Heffner JE (2005) Management of the chronically ventilated patient with a tracheostomy. Chron Respir Dis 2: 151-61.

9. Mungan I, Turan S, Kazancı D, Çilem Bayındır Dicle (2017) Pneumothorax in tracheostomy patient with late onset. Journal of Cardio-Vascular-Thoracic Anaesthesia and Intensive Care Society 23: 149-52.

10. Trouillet JL, Collange O, Belafia F, Blot F, Capellier G (2018) Tracheotomy in the intensive care unit: guidelines from a French expert panel. Ann Intensive Care 8: 37.

11. Putensen C, Theuerkauf N, Guenther U, Vargas M, Pelosi P (2014) Percutaneous and surgical tracheostomy in critically ill adult patients: a meta-analysis. Crit Care 18: 544.

12. Sanabria A (2014) Which percutaneous tracheostomy method is better? A systematic review. Respir Care 59: 1660-70. 